\title{
The Generalized Direct Optimization Technique for Printed Reflectarrays
}

Zhou, Min; Sørensen, Stig Busk; Kim, Oleksiy S.; Jørgensen, Erik; Meincke, Peter; Breinbjerg, Olav; Toso, Giovanni

Published in:

IEEE Transactions on Antennas and Propagation

Link to article, DOI:

10.1109/TAP.2013.2254446

Publication date:

2014

Document Version

Early version, also known as pre-print

Link back to DTU Orbit

Citation (APA):

Zhou, M., Sørensen, S. B., Kim, O. S., Jørgensen, E., Meincke, P., Breinbjerg, O., \& Toso, G. (2014). The Generalized Direct Optimization Technique for Printed Reflectarrays. IEEE Transactions on Antennas and Propagation, 62(4), 1690-1700. https://doi.org/10.1109/TAP.2013.2254446

\section{General rights}

Copyright and moral rights for the publications made accessible in the public portal are retained by the authors and/or other copyright owners and it is a condition of accessing publications that users recognise and abide by the legal requirements associated with these rights.

- Users may download and print one copy of any publication from the public portal for the purpose of private study or research.

- You may not further distribute the material or use it for any profit-making activity or commercial gain

- You may freely distribute the URL identifying the publication in the public portal 


\title{
The Generalized Direct Optimization Technique for Printed Reflectarrays
}

\author{
Min Zhou, Member, IEEE, Stig B. Sørensen, Oleksiy S. Kim, Erik Jørgensen, Member, IEEE, \\ Peter Meincke, Member, IEEE, Olav Breinbjerg, Member, IEEE, and Giovanni Toso, Senior Member, IEEE
}

\begin{abstract}
A generalized direct optimization technique (GDOT) for the design of printed reflectarrays using arbitrarily shaped elements with irregular orientation and position is presented. The GDOT is based on the spectral domain method of moments (SDMoM) assuming local periodicity (LP) and a minimax optimization algorithm. The accuracy of the LP-SDMoM for the design of reflectarrays with irregularly positioned and oriented array elements has been verified by comparisons with full wave method of moments. Three contoured beam reflectarrays, forming a high-gain beam on a European coverage area, have been designed: a broadband design, a circularly polarized design using the variable rotation technique, and a design with irregularly positioned array elements. The latter has been manufactured and measured at the DTU-ESA Spherical Near-Field Antenna Test Facility. An very good agreement between simulated and measured patterns have been obtained, showing accuracies that are comparable to those obtained for conventional shaped reflectors.
\end{abstract}

Index Terms-Accurate antenna analysis, contoured beam, irregular reflectarrays, optimization, reflectarray, satellite antenna.

\section{INTRODUCTION}

$\mathbf{F}$ OR satellite broadcasting and telecommunication applications, the most often used antenna is the shaped reflector antenna. Although this antenna is based on a mature technology, both in terms of manufacturing and simulation tools, it suffers from having large volume and mass, as well as manufacturing cost. In particular the mold depends on the specific antenna requirements and can not be reused for other missions. Printed reflectarrays, on the other hand, consist of a flat surface, they are light, easy and cheap to manufacture, and can be packed more compactly, saving volume during the launch phase. In addition, for a specific coverage area, only the array elements are modified, thus significant recurring costs associated with shaped reflector antennas are avoided. Using printed reflectarrays, low cost, high-gain antennas for space applications can be realized

Manuscript received October 10, 2012; revised December 19, 2012; accepted February 23, 2013. Date of publication March 22, 2013; date of current version April 03, 2014. The reflectarray antenna production and measurement is supported by the European Space Agency (ESTEC contract No.4000101041).

M. Zhou, E. Jørgensen, S. B. Sørensen, and P. Meincke are with the TICRA, DK-1201 Copenhagen, Denmark (e-mail: mz@ticra.com; sbs@ticra.com; ej@ticra.com; pme@ticra.com; ticra@ticra.com).

O. S. Kim and O. Breinbjerg are with the Department of Electrical Engineering, Electromagnetic Systems, Technical University of Denmark, DK-2800 Kgs. Lyngby, Denmark (e-mail: osk@elektro.dtu.dk; ob@elektro.dtu.dk).

G. Toso is with the European Space Agency ESA-ESTEC, 2200 Noordwijk, The Netherlands (e-mail: giovanni.toso@esa.int).

Color versions of one or more of the figures in this paper are available online at http://ieeexplore.iee.org.

Digital Object Identifier 10.1109/TAP.2013.2254446 and they have therefore been the subject of increasing research and development activities [1]-[3].

To obtain a specific far-field pattern with a printed reflectarray, several degrees of freedom can be used, e.g., the size [4], [5], the shape [6]-[9], the orientation [10], [11], and the position [12], [13] of the array elements. An accurate and efficient design procedure, capable of including all these parameters, is a challenging task. Recently, the European Space Agency (ESA) has promoted activities to improve and extend the analysis and synthesis procedures for reflectarrays including all the degrees of freedom [14]-[16].

The conventional approach for the design of contoured/multi beam reflectarrays uses a phase-only optimization technique (POT) [17], involving two steps (for pencil beam reflectarrays, the first step is skipped); first, a phase-only synthesis determines the phase distribution on the reflectarray surface; second, the array elements are adjusted, element by element, to comply with the synthesized phase distribution. Several contoured beam reflectarrays have been designed using this technique [17]-[19]. The POT is efficient since the analysis of all array elements at each iteration is avoided. However, a direct optimization technique, where all the array elements are simultaneously optimized, tends to produce improved designs. Such a direct technique was presented in [15], where several contoured beam reflectarrays were designed and compared to similar designs obtained using the POT. The comparisons showed that the designs obtained using the direct optimization technique are superior in performance, both for multi-frequency and dual-polarization designs.

The direct optimization technique reported in [15] is meant for reflectarrays where the array elements are located in a regular grid. Furthermore, only the size of square patches was used as a degree of freedom. Reflectarrays with even better performance may be designed if additional degrees of freedom, e.g., the position and orientation of the array elements, are included in the optimization. Such a technique was presented in [13], wherein the array elements were located in a strongly irregular grid and the analysis of each element was performed using a full-wave method of moments (MoM) that included the nearest neighboring elements. Thus, the overall synthesis was very time consuming.

In this work, we generalize the direct optimization technique of [15] to include several degrees of freedom. These are the position and orientation as well as size and shape parameters of printed reflectarray elements. The generalized direct optimization technique (GDOT) is based on the spectral domain method 
of moments (SDMoM) assuming local periodicity (LP) [5]. The use of this technique for the design and analysis of reflectarrays with irregularly positioned and oriented array elements is new, and we show that the LP-SDMoM is sufficiently accurate to analyze and optimize reflectarrays based on arbitrarily shaped elements with irregular position and orientation.

Three contoured beam reflectarrays forming a high-gain beam on a European coverage area have been designed to illustrate the capabilities of the GDOT: a broadband dual linearly polarized design, a circularly polarized design based on the variable rotation technique [10], and a linearly polarized design with irregularly positioned array elements. The latter has been manufactured at the Technical University of Denmark (DTU) and measured at the DTU-ESA Spherical Near-Field Antenna Test Facility [20]. The agreement between simulations and measurements is very good, thus verifying the accuracy of the GDOT.

This paper is organized as follows. Section II describes the GDOT. The reflectarray designs are described in Section III. In Section IV, simulations are compared to the measurements, and conclusions are given in Section V.

\section{Generalized Direct Optimization Technique}

In this section, the analysis and optimization methods used in the direct optimization technique are reviewed and generalized to include the position as well as the orientation of arbitrarily shaped and sized array elements into the optimization.

\section{A. Analysis and Optimization Methods}

The GDOT uses the same optimization algorithm that is used in the TICRA software packages POS [21] and CHAMP [22]. It is based on a gradient minimax algorithm [23] for non-linear optimization.

The far-field objectives for the reflectarray are specified in a number of far-field points in the $(u, v)$-plane where $u=\sin \theta \cos \phi, v=\sin \theta \sin \phi$, and $(\theta, \phi)$ the standard spherical coordinates. At each optimization iteration, the maximum difference between realized and specified objectives is minimized. The optimization variables are the geometrical parameters of the array elements, e.g., the size, orientation, and position of the array element. Both co- and cross-polar radiation patterns can be optimized for multiple frequencies, polarizations, and feed illuminations.

For the far-field calculations, two techniques are considered, the Floquet harmonics technique [24, Technique II], and the continuous spectrum technique [24, Technique III]. Whereas the continuous spectrum technique is slightly more accurate, the Floquet harmonics technique is more efficient. Thus, the Floquet harmonics technique is used to calculate the far-field during the optimization, whereas the continuous spectrum technique is used to evaluate the final optimized reflectarray.

The Floquet harmonics technique is based on the field equivalence principle $[25$, p. 106] and uses the scattering matrices to calculate the equivalent currents. The scattering matrix $\bar{S}$ for a single array element is defined as

$$
\mathbf{E}^{\mathrm{s}}=\bar{S} \cdot \mathbf{E}^{\mathrm{i}}
$$

and is calculated from the fundamental Floquet harmonic through the LP-SDMoM formulation. Herein, $\mathbf{E}^{\mathrm{s}}$ and $\mathbf{E}^{\mathrm{i}}$ are the scattered and incident plane wave, respectively. To ensure an accurate and efficient calculation of the scattering matrices, higher-order hierarchical Legendre basis functions [26] are used to model the electric currents on the array elements. For canonically shaped array elements e.g., rectangular patches, singular entire domain basis functions with the correct edge conditions, reproducing the singularities of the electric current at the edges of the array elements, have proven to yield accurate results [27]. It is shown in [28] that the higher-order hierarchical Legendre basis functions can be applied to any arbitrarily shaped array elements, and are at the same time capable of yielding results of the same accuracy as those obtained using singular basis functions. The versatility of the higher-order hierarchical Legendre basis functions is a key feature in the GDOT as it enables the optimization of reflectarrays consisting of non-canonical element shapes, e.g., concentric square/ring loops, phoenix elements, and many others [6]-[9]. In this work, we consider several element shapes, namely square patches, concentric square loops, square loop/patch combination, and triple dipoles.

To avoid the calculation of the scattering matrices of all array elements at each optimization iteration, the scattering matrices can be calculated in advance and stored in a look-up table [13], [18], which is accessed during the optimization by means of local cubic interpolation [29, Chap. 25]. For a given frequency, dielectric substrate, and unit-cell size, the scattering matrix depends on the illumination angles $\theta^{\mathrm{i}}$ and $\phi^{\mathrm{i}}$ (see Fig. 1), and the geometry of the array element. For the cases we have considered, a sufficient accuracy can be obtained by using approximately $N_{\theta}=12$ sample values in $\theta^{\mathrm{i}}$. The variation of the scattering coefficients in $\phi^{\mathrm{i}}$ is $2 \pi$-periodic and can thus be represented by a finite Fourier series expansion

$$
\bar{S}\left(\theta^{\mathrm{i}}, \phi^{\mathrm{i}}\right)=\sum_{s=-N_{\mathrm{m}}}^{N_{\mathrm{mm}}} \bar{c}_{s}\left(\theta^{\mathrm{i}}\right) e^{j s \phi^{\mathrm{i}}}
$$

where [30, App. A4]

$$
\bar{c}_{s}=\frac{\Delta \phi}{2 \pi} \sum_{p=1}^{2 N_{\mathrm{m}}+1} \bar{S}\left(\theta^{\mathrm{i}}, \phi_{p}^{\mathrm{i}}\right) e^{-j s \phi_{p}^{\mathrm{i}}} .
$$

Herein, $\Delta \phi=2 \pi /\left(2 N_{\mathrm{m}}+1\right)$ and $\phi_{p}^{\mathrm{i}}=\Delta \phi(p-1)$. We have observed, for the reflectarrays presented in this paper, that $N_{\mathrm{m}}=2$ is adequate, yielding a total of only $N_{\phi}=5$ sample values in $\phi^{\mathrm{i}}$. For the variation of the scattering coefficients due to the different element sizes, approximately $N_{\text {elements }}=60$ is sufficient. Thus for a given frequency, substrate, and unit-cell size, the total number of full-wave computations of $\bar{S}$ needed in the look-up table to obtain an accurate interpolation is $N_{\text {total }}=$ $N_{\theta} N_{\phi} N_{\text {elements }}=12 \cdot 5 \cdot 60=3600$. This can be computed within a couple of minutes on a standard laptop computer. The look-up table can be reused and needs only to be recalculated if other substrates, frequencies, or unit-cell sizes are used.

For more details on the analysis methods and the look-up table, the reader is referred to [15]. 


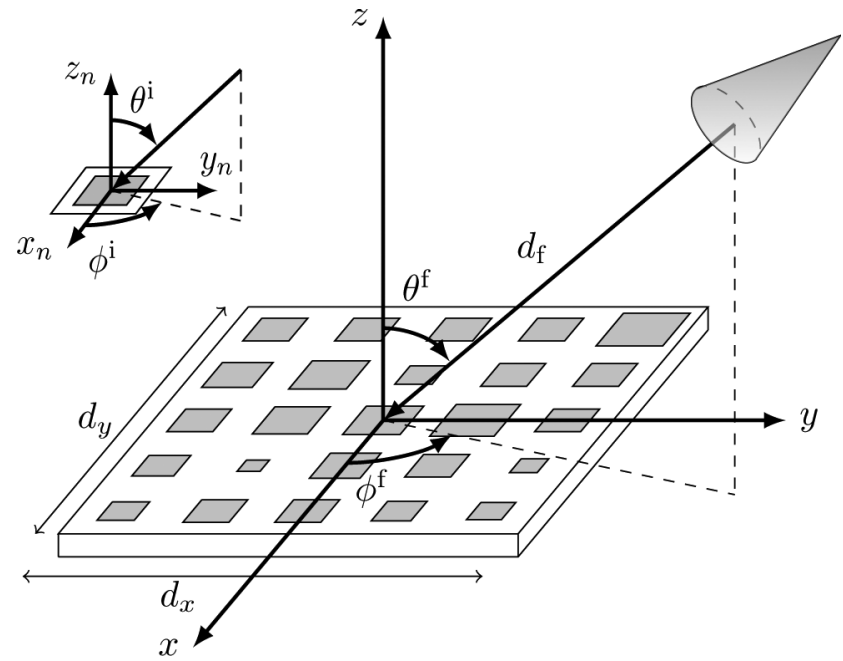

Fig. 1. Reflectarray (bottom) and unit-cell (top left) parameters.

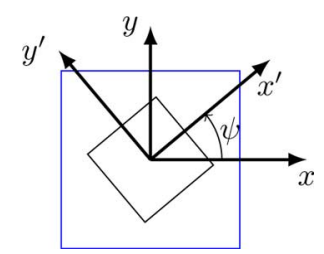

Fig. 2. A rotated square patch in a unit-cell, where the rotation angle is denoted by $\psi$.

\section{B. Irregularly Orientated Array Elements}

In order to exploit the orientation of the array elements in the GDOT, the look-up table has to be extended to include also the rotation angle $\psi$ of the array element, see Fig. 2.

The rotation angle $\psi$ is $2 \pi$-periodic and hence the variation of the scattering matrices in $\psi$ can be represented by a finite Fourier series expansion. Thus, the equation in (2) is replaced by

$$
\bar{S}\left(\theta^{\mathrm{i}}, \phi^{\mathrm{i}}, \psi\right)=\sum_{s=-N_{\mathrm{m}}}^{N_{\mathrm{mI}}} \sum_{r=-N_{\mathrm{n}}}^{N_{\mathrm{rI}}} \bar{c}_{s r}\left(\theta^{\mathrm{i}}\right) e^{j s \phi^{\mathrm{i}}} e^{j r \psi}
$$

The Fourier coefficients $c_{s r}$ are given by

$$
\bar{c}_{s r}=\frac{\Delta \phi \Delta \psi}{4 \pi^{2}} \sum_{p=1}^{2 N_{\mathrm{m}}+1} \sum_{q=1}^{2 N_{\mathrm{n}}+1} \bar{S}\left(\theta^{\mathrm{i}}, \phi_{p}^{\mathrm{i}}, \psi_{q}\right) e^{-j s \phi_{p}^{\mathrm{i}}} e^{-j r \psi_{q}}
$$

where $\Delta \psi=2 \pi /\left(2 N_{\mathrm{n}}+1\right)$ and $\psi_{q}=\Delta \psi(q-1)$. The value of $N_{\mathrm{n}}$ depends on the shape and size of the array element. For the elements presented in this work, $N_{\mathrm{n}}=5$, giving a total $N_{\psi}=11$ sample values, is sufficient for an accurate representation of the variation in $\psi$. The total number of scattering matrix samples needed in the look-up table is now $N_{\text {total }}=$ $N_{\theta} N_{\phi} N_{\text {elements }} N_{\psi}=12 \cdot 5 \cdot 60 \cdot 11=39600$ per frequency. The derivatives with respect to $\psi$, which are required in the optimization, are readily obtained analytically by differentiation of (4).

\section{Irregularly Positioned Array Elements}

In order to utilize the position of the array elements in the GDOT, an irregular distribution of element positions is obtained through a mapping from a regular to an irregular grid by adding a distortion to the regular grid.

Let us define $(\alpha, \beta)$ as normalized coordinates in the regular grid such that $|\alpha| \leq 1$ and $|\beta| \leq 1$. Then, the normalized coordinates in the irregular grid are given by $\left(\alpha^{\prime}, \beta^{\prime}\right)=$ $\left(\alpha+d_{\alpha}, \beta+d_{\beta}\right)$, where the distortion functions are

$$
\begin{aligned}
& d_{\alpha}(\alpha, \beta)=(\alpha-1)(\alpha+1) \sum_{p=0}^{P} \sum_{q=0}^{Q} c_{p q}^{\alpha} T_{p}(\alpha) T_{q}(\beta) \\
& d_{\beta}(\alpha, \beta)=(\beta-1)(\beta+1) \sum_{p=0}^{P} \sum_{q=0}^{Q} c_{p q}^{\beta} T_{p}(\alpha) T_{q}(\beta)
\end{aligned}
$$

Herein, $T_{i}$ is the Chebyshev polynomial of order $i$, and $c_{p q}^{\alpha}$ and $c_{p q}^{\beta}$ are the distortion coefficients. In contrast to [13], where the edges of the reflectarray are not constrained, the terms in front of the summations ensure that the edges of the reflectarray are kept fixed to avoid any undesired increase in antenna size introduced by the mapping.

The degree of the distortion is determined by the values of $c_{p q}^{\alpha}$ and $c_{p q}^{\beta}$ and the maximum polynomial order $P$ and $Q$. To avoid distortions with overlapping array elements, upper and lower bounds are specified for $c_{p q}^{\alpha}$ and $c_{p q}^{\beta}$, and the polynomial order should not exceed 4 . Only a few, 2 to 6 , distortion coefficients are needed to achieve strong irregularities, as shown in Section II-E. The distortion coefficients $c_{p q}^{\alpha}$ and $c_{p q}^{\beta}$ are the variables used to optimize the positions of the array elements. In this way, the optimization of the individual array element position, which can be rather complicated, is avoided.

Unlike the other optimization variables, the derivatives with respect to $c_{p q}^{\alpha}$ and $c_{p q}^{\beta}$ can not be determined analytically since a change in $c_{p q}^{\alpha}$ or $c_{p q}^{\beta}$ affects all array elements. As a result, the derivatives are computed numerically by finite difference approximations at the cost of higher computation time.

\section{Analysis of the Distorted Cell}

Due to the grid distortion, the array elements are positioned in a non-periodic lattice and the LP-SDMoM can not be directly applied. Therefore, an equivalent unit-cell has to be defined to approximate locally each distorted cell. The center of the array element within the distorted cell is positioned at the intersection of the two diagonal lines of the distorted cell, see Fig. 3(a). Let us now define an equivalent square cell with the same area as the distorted cell. The equivalent cell has the same diagonal intersection as the distorted cell and is oriented in parallel with the bisector lines of the two diagonal lines of the distorted cell. This is illustrated in Fig. 3(b), where the bisector lines are shown by dashed lines. The equivalent cell is used in the LP-SDMoM to calculate the scattering matrices.

The analysis procedure for reflectarrays with irregularly positioned elements is now the same as for those with regularly positioned elements, except that the unit-cells of the array elements are of different sizes. As a result, the look-up table has to be further extended to include also samples of different sized unit-cells. The number of unit-cell samples needed depends on 


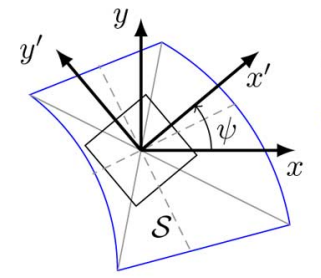

(a)

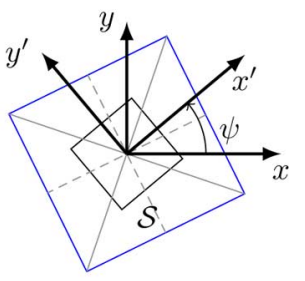

(b)
Fig. 3. An example of (a) a distorted cell and (b) its equivalent square cell. Both cells have the area $\mathcal{S}$. The center of the patch is located at the intersection of the two solid diagonal lines of the distorted cell. The rotation of the patch is given by the rotation angle $\psi$ with respect to the coordinate system of the regular grid.

the degree of the grid distortion. For the results presented in this paper, $N_{\text {cell }}=50$ samples are sufficient, yielding a look-up table that requires $N_{\text {total }}=N_{\theta} N_{\phi} N_{\text {elements }} N_{\psi} N_{\text {cell }}=12 \cdot 5$. $60 \cdot 11 \cdot 50=1980000$ scattering matrix calculations per frequency. The computation time on a standard laptop is several hours, which is a significant increase compared to the regular array case. It is, however, still acceptable since the look-up table only needs to be calculated once prior to the optimization.

\section{E. Analysis Accuracy}

The use of the LP-SDMoM for the design and analysis of reflectarrays with irregularly positioned and oriented array elements is new, and thus the accuracy of the technique for such reflectarrays has to be established.

To this end, two offset pencil beam reflectarrays with different distortions have been designed, the first design with the beam towards the specular direction and the second with the beam towards the broadside direction. The distorted grids are kept fixed and only the size and orientation of the array elements are optimized. The values of $c_{p q}^{\alpha}$ and $c_{p q}^{\beta}$ are selected empirically to ensure a strong but realistic distortion. The feed is a linear polarized Gaussian beam with a taper of $-15 \mathrm{~dB}$ at $30^{\circ}$. The geometrical parameters are summarized in Table I with respect to the coordinate system shown in Fig. 1. The mask layouts of the optimized reflectarrays are shown in Fig. 4. The number of distortion coefficients for the specular and broadside cases are 2 and 6 , respectively.

A full wave MoM is used as reference. For the calculation of the currents on the array elements, the MoM relies on the spatial dyadic Green's function for a grounded dielectric slab [31], thus assuming infinite substrate and ground plane.

The radiation patterns for the two reflectarrays calculated using LP-SDMoM and MoM are shown in Fig. 5. The continuous spectrum technique is used to calculate the far-field in both methods, thus accounting for the finite size of the reflectarrays [24]. A very good agreement between the two methods is observed. For the specular case, the predicted peak directivity using both methods is $30.7 \mathrm{dBi}$, whereas for the broadside case, LP-SDMoM and MoM yield $31.1 \mathrm{dBi}$ and $31.0 \mathrm{dBi}$, respectively. Also the cross-polar radiation levels are extremely close; the deviations are around $1-3 \mathrm{~dB}$ at $-40 \mathrm{~dB}$ below the co-polar peak. Several irregular designs have been optimized and analyzed using both LP-SDMoM and MoM, and it was observed that the LP-SDMoM is accurate in all cases.

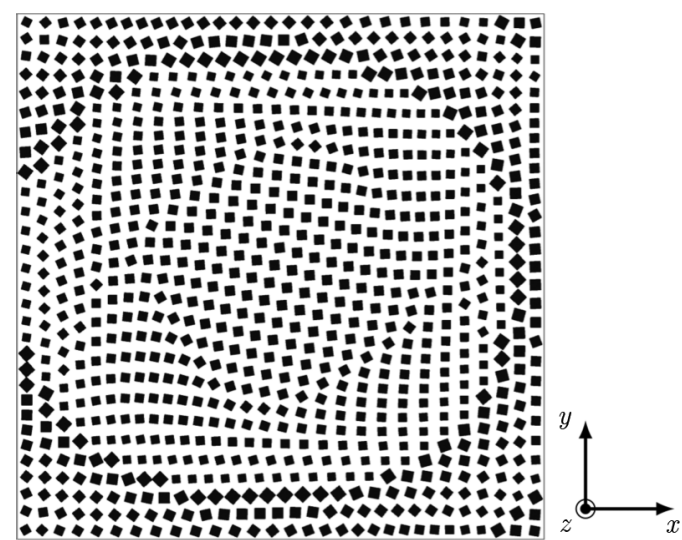

(a)

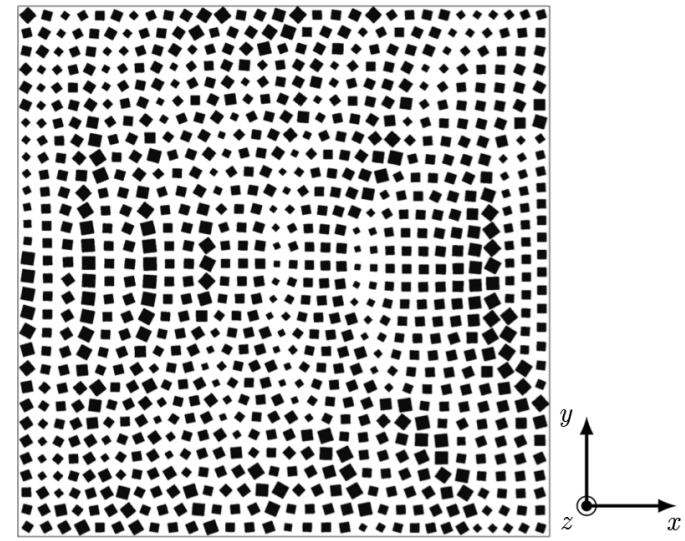

(b)

Fig. 4. Pencil beam reflectarrays with irregularly positioned and oriented array elements. The reflectarrays are designed to radiate a pencil beam towards (a) the specular direction and (b) the broadside direction.

TABLE I

Pencil Beam Reflectarray Data

\begin{tabular}{l|c}
\hline \hline Center frequency & $10 \mathrm{GHz}$ \\
\hline Reflectarray dimensions & $405 \mathrm{~mm} \times 405 \mathrm{~mm}$ \\
\hline Number of elements & $30 \times 30$ \\
\hline Relative permittivity & $\epsilon_{\mathrm{r}}=3.66$ \\
\hline Loss tangent & $\tan \delta=0.0037$ \\
\hline Substrate thickness & $h=1.524 \mathrm{~mm}$ \\
\hline Feed distance to center of array & $d_{\mathrm{f}}=0.6 \mathrm{~m}$ \\
\hline Feed offset angle & $\theta^{\mathrm{f}}=30^{\circ}, \phi^{\mathrm{f}}=0^{\circ}$ \\
\hline
\end{tabular}

The good accuracy of the LP-SDMoM, despite the irregularities, can be attributed to the systematic manner in which the grids are distorted by (6). Although the periodicity assumption is violated due to the different cell and element sizes as well as the irregular positions, the change in cell size and position is rather smooth and the errors introduced by the periodicity assumption are small.

\section{REFLECTARRAY DESIGN}

In this section, we design several offset contoured beam reflectarrays forming a high-gain beam on a European coverage area with the aim to demonstrate the capabilities of the GDOT. Three reflectarrays are designed: a dual linearly polarized broadband design, a circularly polarized design based on the variable rotation technique [10], and a linearly polarized design 


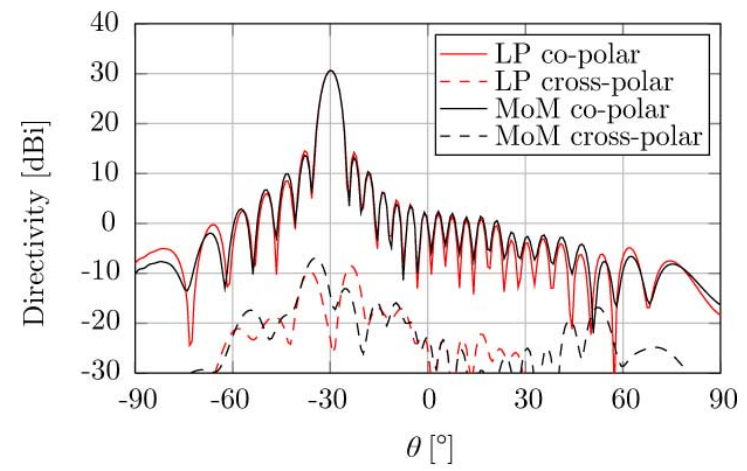

(a)

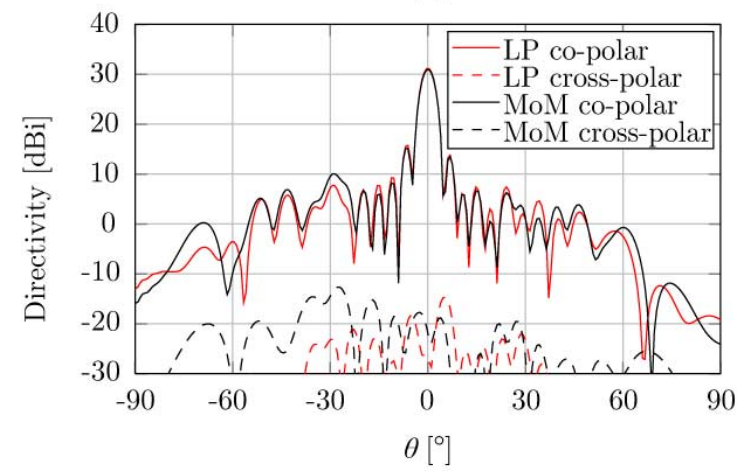

(b)

Fig. 5. Comparison of the radiation pattern at $\phi=0$ calculated using LP-SDMoM and MoM for (a) the specular radiation case and (b) the broadside radiation case.

with irregularly positioned array elements. The reflectarray parameters are the same as those listed in Table I, except that the dimensions of the designs are $600 \times 600 \mathrm{~mm}^{2}$, corresponding to approximately $20 \times 20 \lambda_{0}^{2}$, with $\lambda_{0}$ being the free space wavelength, at the center frequency $10 \mathrm{GHz}$. In addition, the substrate thickness is different for the three designs.

Although some measures are taken during the design process to ensure a good antenna performance, e.g., by selecting appropriate array elements, the purpose of these designs is not to obtain the best possible reflectarray but to illustrate the capabilities of the GDOT to yield an optimum design within a given set of parameters.

\section{A. Broadband Design}

The goal of this design (Design A) is to maximize the directivity within the European coverage area and at the same time minimize the cross-polar radiation within the same coverage area in the frequency range $9-11 \mathrm{GHz}$ for two linear polarizations, $\mathrm{V}$ - and H-polarization. H-polarization is in the offset plane ( $x z$-plane in Fig. 1) and V-polarization in the orthogonal plane.

For broadband performance, the variation of the phase of the scattering coefficient as function of the geometrical parameters should be slow and almost parallel at different frequencies [1], [7], [9]. The concentric square loops have been demonstrated to have good phase responses that provide a good bandwidth [6], [7]. They have several parameters that can be adjusted to control the phase of the scattering coefficient: the lengths and

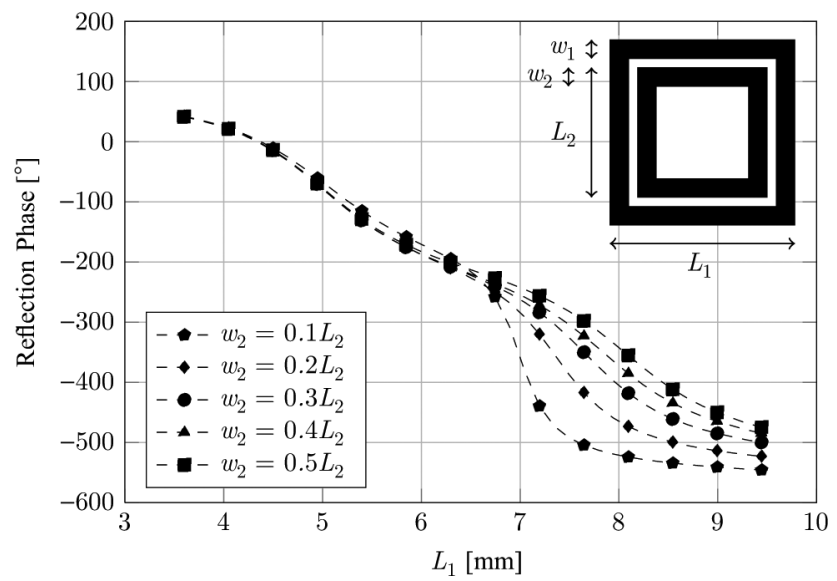

Fig. 6. The phase of the scattering coefficient of concentric square loops in a periodic environment as function of the outer loop length $L_{1}$ for different inner loop widths $w_{2}$. The inner loop length is $L_{2}=0.75 L_{1}$, the width of the outer loop is $w_{1}=0.075 L_{1}$.

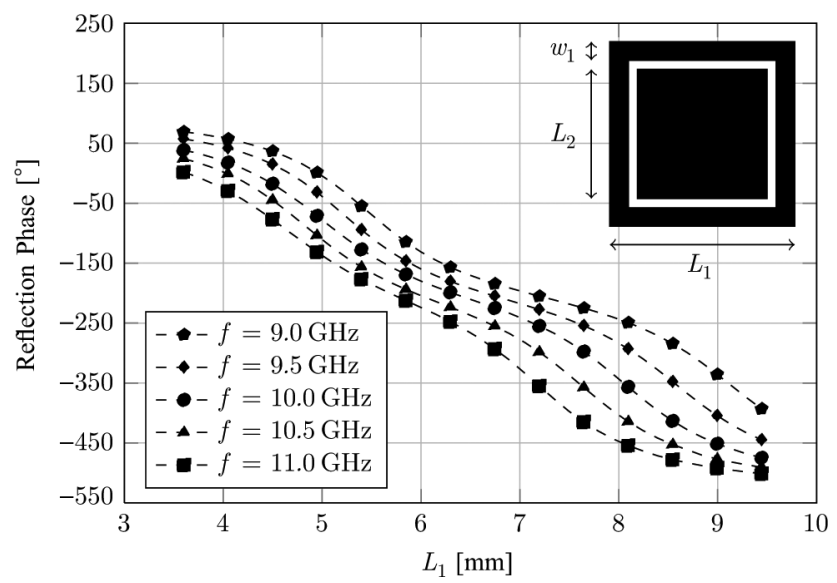

Fig. 7. The phase of the scattering coefficient of a square loop/patch combination element in a periodic environment as function of the outer loop length $L_{1}$ for different frequencies. The inner loop length is $L_{2}=0.75 L_{1}$, the width of the outer loop is $w_{1}=0.075 L_{1}$.

widths of the outer and inner loops. In Fig. 6, the phase of the scattering coefficient of concentric square loops in a periodic environment for different widths of the inner loop $w_{2}$ is shown. The phase is calculated at $10 \mathrm{GHz}$ under normal plane wave incidence and displayed as a function of the length of the outer loop $L_{1}$. The substrate thickness is $h=3.048 \mathrm{~mm}$ and the size of the square unit-cell is $d=10.5 \mathrm{~mm}$. Based on a number of simulations, the width of the outer loop is $w_{1}=0.075 L_{1}$ and the length of the inner loop is $L_{2}=0.75 L_{1}$. This is to ensure a slow phase response versus $L_{1}$ and at the same time maintain a phase variation over $360^{\circ}$, which is required for the design of reflectarrays. It is seen in Fig. 6 that the slope of the phase curve decreases for increasing $L_{1}$ when $w_{2}$ is wide. The case where $w_{2}=0.5 L_{2}$ is equivalent to the case where the inner loop is replaced by a square patch. Here, the reflection phase varies slowly as function of $L_{1}$. The phase response as function of $L_{1}$ for different frequencies between $9-11 \mathrm{GHz}$ is shown in Fig. 7. It is seen that the phase curves versus $L_{1}$ are close to being parallel at the different frequencies. A similar result has been observed in [32]. Due to these properties, this square loop/patch 




Fig. 8. The mask layout of Design A.

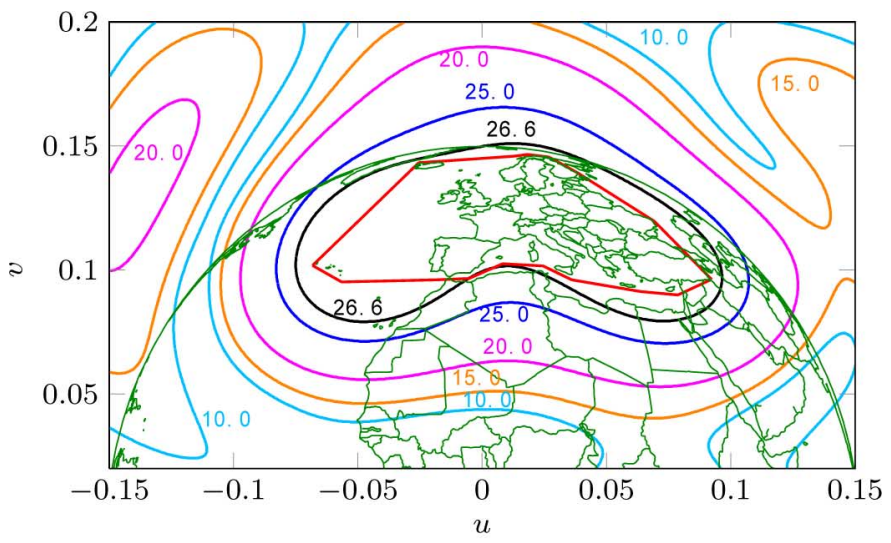

(a)

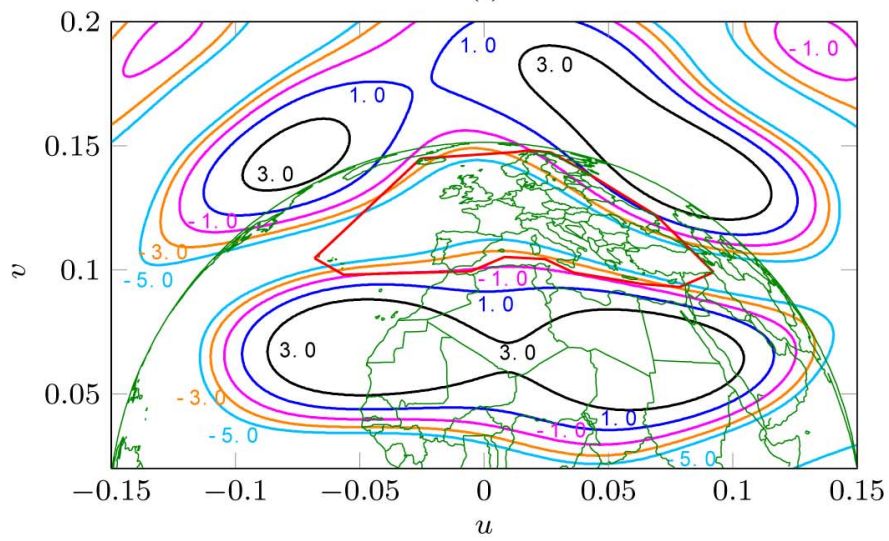

(b)

Fig. 9. Simulated radiation patterns of Design A for H-polarization at $10 \mathrm{GHz}$, (a) co-polar pattern and (b) cross-polar pattern. The European coverage area seen from the longitude $0^{\circ}$ geostationary orbital position is shown as the red polygon.

combination is used in Design A. In the optimization, $w_{1}$ and $L_{2}$ are fixed according to Fig. 7 and only $L_{1}$ is optimized. A scattering matrix look-up table for frequencies $f=9,10$, and $11 \mathrm{GHz}$ has been calculated for this design.

As a starting point of the optimization, identical elements are used. A Gaussian beam with a taper of $-15 \mathrm{~dB}$ at $30^{\circ}$ is used as a feed. The optimized reflectarray consists of $57 \times 57$ elements and the mask layout of the design is shown in Fig. 8. The radiation pattern at $10 \mathrm{GHz}$ for H-polarization is shown in Fig. 9, and it shows that a minimum directivity of $26.6 \mathrm{dBi}$ is obtained
TABLE II

Performance of Dual-polarized Broadband Reflectarkay Design

\begin{tabular}{ccccc}
\hline \hline & \multicolumn{2}{c}{ H-polarization } & \multicolumn{2}{c}{ V-polarization } \\
\hline $\begin{array}{c}\text { Frequency } \\
(\mathrm{GHz})\end{array}$ & $\begin{array}{c}\text { Minimum } \\
\text { Directivity } \\
(\mathrm{dBi})\end{array}$ & $\begin{array}{c}\text { Minimum } \\
\text { XPD } \\
(\mathrm{dB})\end{array}$ & $\begin{array}{c}\text { Minimum } \\
\text { Directivity } \\
(\mathrm{dBi})\end{array}$ & $\begin{array}{c}\text { Minimum } \\
\text { XPD } \\
(\mathrm{dB})\end{array}$ \\
\hline 8.5 & 24.7 & 27.1 & 24.8 & 28.1 \\
9.0 & 26.6 & 26.7 & 26.5 & 26.6 \\
9.5 & 26.4 & 26.4 & 26.4 & 26.9 \\
10.0 & 26.6 & 26.4 & 26.5 & 27.6 \\
10.5 & 26.5 & 25.0 & 26.4 & 27.4 \\
11.0 & 26.6 & 24.1 & 26.4 & 28.4 \\
11.5 & 24.9 & 22.9 & 25.1 & 26.3 \\
\hline \hline
\end{tabular}

within the European coverage area and that the cross-polar radiation has been successfully suppressed to below $1 \mathrm{dBi}$ within the same coverage area.

The performance of the design is summarized in Table II. It is seen that the minimum directivity is above $26.4 \mathrm{dBi}$ for both polarizations between $9-11 \mathrm{GHz}$ and drops to approximately $25 \mathrm{dBi}$ at $8.5 \mathrm{GHz}$ and $11.5 \mathrm{GHz}$, which are outside of the desired frequency range. This shows that the reflectarray has been successfully optimized to operate in the specified frequency range of $20 \%$ bandwidth.

In this design, only $L_{1}$ has been optimized, while $w_{1}$ and $L_{2}$ were fixed. It is expected that better performances can be obtained if $w_{1}$ and $L_{2}$ are also included as optimization variables.

\section{B. Circularly Polarized Design}

In this design (Design B), a right hand circularly polarized (RHCP) reflectarray that radiates a high-gain beam on the European coverage area in the frequency range $9-11 \mathrm{GHz}$ is realized by utilizing the variable rotation technique (VRT) [10], [11]. The VRT uses identical array elements with different angular rotations to achieve a given far-field beam. Suppose an array element is illuminated by a circularly polarized incident wave, then by rotating the array element by angle $\psi$ (Fig. 2), the phase of the scattered field of the array element is shifted by $2 \psi$. Thus, by adjusting the rotation angles of all array elements, a given phase distribution can be realized to radiate a specified far-field beam [10].

It is shown in [10], and explicitly stated in [33], that the phase of the scattering coefficient for two orthogonal linear polarizations, e.g., $\mathrm{V}$ - and H-polarization, has to be different by $180^{\circ}$, so that the scattered field has the same sense of circular polarization as the incident wave with a phase shift of $\pm 2 \psi$ depending on the polarization of the incident wave. Thus, by selecting an array element that can realize the $180^{\circ}$ phase difference in a wide frequency range is the key for the use of the VRT.

In this design, we use the triple dipole element [34] as the array element. In Fig. 10, the phase difference between V-and $\mathrm{H}$-polarization of the triple dipole element is shown for different frequencies as function of the center dipole length $L$. The length of the two parallel dipoles is $L_{2}=0.65 \mathrm{~L}$, and the width of each dipole is $w=0.1 L$. The substrate thickness is $h=3.5 \mathrm{~mm}$ and the unit-cell size $d=12 \mathrm{~mm}$. The phase is calculated for a normally incident plane wave and shown for $f=9,10,11 \mathrm{GHz}$. Based on the phase difference, a dipole length of $L=8.5 \mathrm{~mm}$ has been selected for the optimization, so that a phase difference of $180^{\circ}$ between $\mathrm{V}$ - and H-polarization is achieved at $10 \mathrm{GHz}$. 


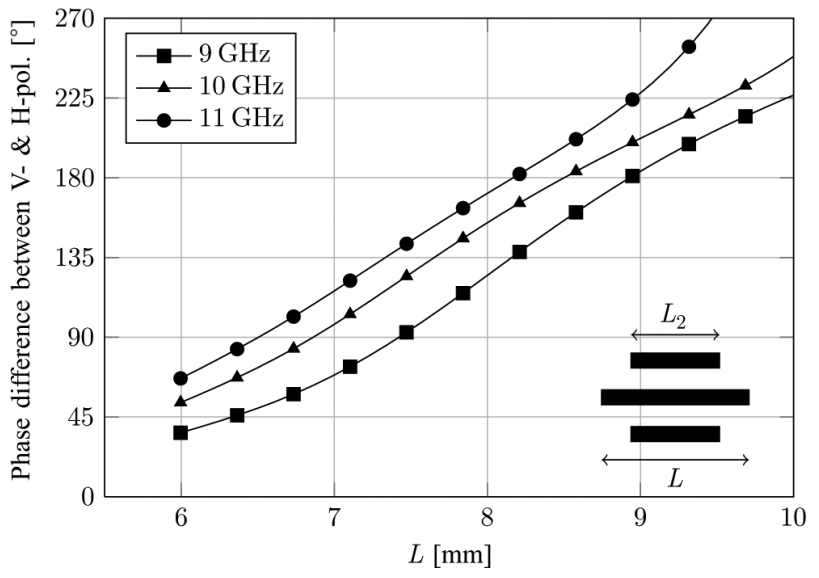

Fig. 10. The phase difference between $\mathrm{V}$ - and $\mathrm{H}$-polarization as function of the center dipole length $L$. The lengths of the two parallel dipoles are both $L_{2}=$ $0.65 \mathrm{~L}$. The widths of the dipoles are $w=0.1 \mathrm{~L}$.

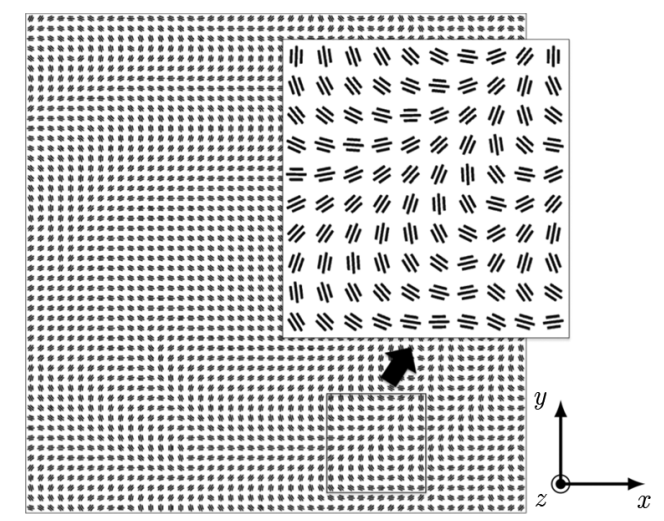

Fig. 11. The mask layout of Design B.

TABLE III

Performance of Circularly Polarized Reflectarray Design

\begin{tabular}{ccccc}
\hline \hline & \multicolumn{2}{c}{ 20\% design } & \multicolumn{2}{c}{$10 \%$ design } \\
\hline $\begin{array}{c}\text { Frequency } \\
(\mathrm{GHz})\end{array}$ & $\begin{array}{c}\text { Minimum } \\
\text { Directivity } \\
(\mathrm{dBi})\end{array}$ & $\begin{array}{c}\text { Minimum } \\
\text { XPD } \\
(\mathrm{dB})\end{array}$ & $\begin{array}{c}\text { Minimum } \\
\text { Directivity } \\
(\mathrm{dBi})\end{array}$ & $\begin{array}{c}\text { Minimum } \\
\text { XPD } \\
(\mathrm{dB})\end{array}$ \\
\hline 8.5 & 25.0 & 16.4 & 22.6 & 15.2 \\
9.0 & 26.6 & 24.7 & 25.3 & 24.1 \\
9.5 & 26.7 & 23.0 & 26.6 & 24.0 \\
10.0 & 26.8 & 20.7 & 26.7 & 24.1 \\
10.5 & 26.8 & 20.5 & 26.7 & 27.0 \\
11.0 & 26.7 & 17.3 & 25.3 & 21.5 \\
11.5 & 25.1 & 14.8 & 23.9 & 12.2 \\
\hline \hline
\end{tabular}

At $9 \mathrm{GHz}$ and $11 \mathrm{GHz}$, the phase difference changes to approximately $155^{\circ}$ and $197^{\circ}$, respectively. The specified bandwidth of $20 \%$ is rather large and the triple dipole element does not provide the necessary phase difference of $180^{\circ}$ in the entire frequency range. However, it has significantly better performance compared to more simple elements e.g., single dipoles.

In this design, only the rotation angles of the triple dipoles are optimized. No cross-polar suppression has been specified for the optimization of this design. As the starting point of the optimization, all the dipoles are oriented with $\psi=0$. A RHCP Gaussian beam with a taper of $-15 \mathrm{~dB}$ at $30^{\circ}$ is used as feed. The optimized reflectarray consists of $50 \times 50$ elements and the mask layout is shown in Fig. 11. It is seen in Table III,

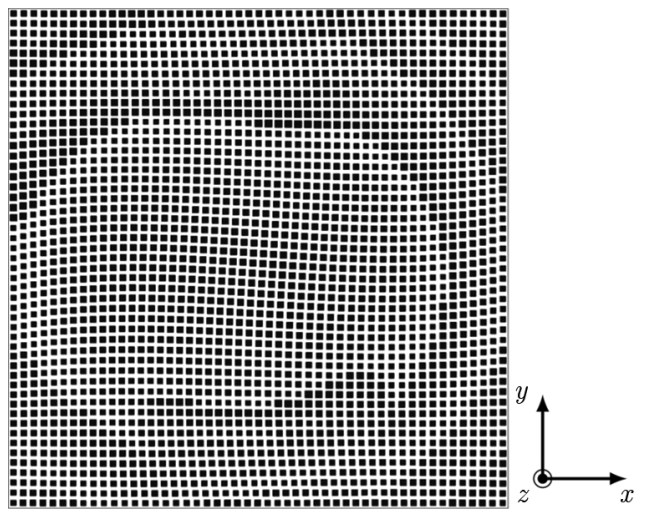

Fig. 12. The mask layout of Design C.

where the performance of the design ( $20 \%$ design) in the frequency range $8.5-11.5 \mathrm{GHz}$ is listed, that a minimum directivity within the European coverage area above $26.6 \mathrm{dBi}$ is achieved in the frequency range $9-11 \mathrm{GH} \%$. The minimum directivity decreases outside the specified frequency range, as expected.

The minimum XPD within the frequency range is relatively low with the best value of $24.7 \mathrm{~dB}$ at $9 \mathrm{GHz}$. In the attempt to reduce the cross-polar radiation, a design with cross-polar suppression has also been optimized. However, approximately $2 \mathrm{~dB}$ in the minimum directivity was lost, and the cross-polar radiation was only suppressed to a minimum XPD of approximately $20 \mathrm{~dB}$ in the desired frequency range. This is a direct consequence of the large bandwidth specified in the optimization. A similar design including cross-polar suppression for a bandwidth of $10 \%(9.5-10.5 \mathrm{GHz})$ was also optimized and the performance is listed in Table III (10\% design). A minimum directivity of $26.6 \mathrm{dBi}$ in the specified frequency range is maintained, but the minimum XPD has been improved to $24.0 \mathrm{~dB}$. This shows that the VRT is rather sensitive with respect to the required $180^{\circ}$ phase difference, particularly regarding the cross-polar radiation.

It is expected that better performance can be achieved if an array element with $180^{\circ}$ phase difference between $\mathrm{V}$ - and H-polarization in a wider frequency range can be found.

\section{Irregularly Positioned Design}

In this design (Design C), we consider a reflectarray with irregularly positioned array elements. The reflectarray radiate a high-gain beam within the European coverage area with crosspolar suppression within the same area and sidelobe suppression within a southern African contour (see Fig. 13). The reflectarray consists of $50 \times 50$ array elements and is optimized for $\mathrm{H}$-polarization and only at $10 \mathrm{GHz}$. The substrate thickness is $h=0.762 \mathrm{~mm}$. A corrugated horn with a taper of $-17.5 \mathrm{~dB}$ at $30^{\circ}$ at $10 \mathrm{GHz}$ is used as feed. The feed has been measured at the DTU-ESA Spherical Near-Field Antenna Test Facility, and this feed pattern is used in the optimization. Square patches are used as array elements and the patch sizes are the optimization parameters. In addition to the patch sizes, 10 grid distortion coefficients are included in the optimization.

The mask layout of the optimized reflectarray is shown in Fig. 12. Simulations show a minimum directivity of $27.3 \mathrm{dBi}$ 


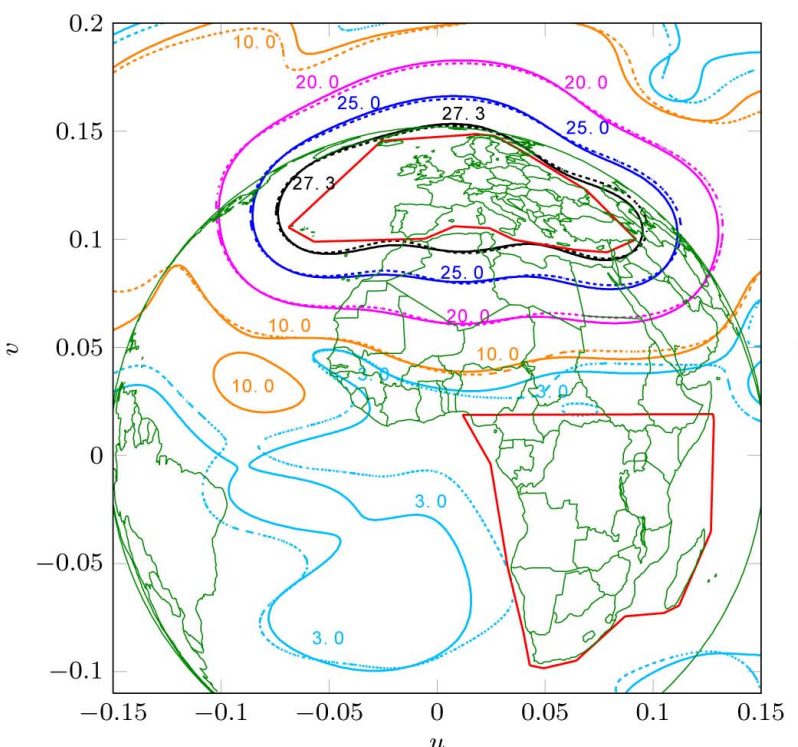

(a)

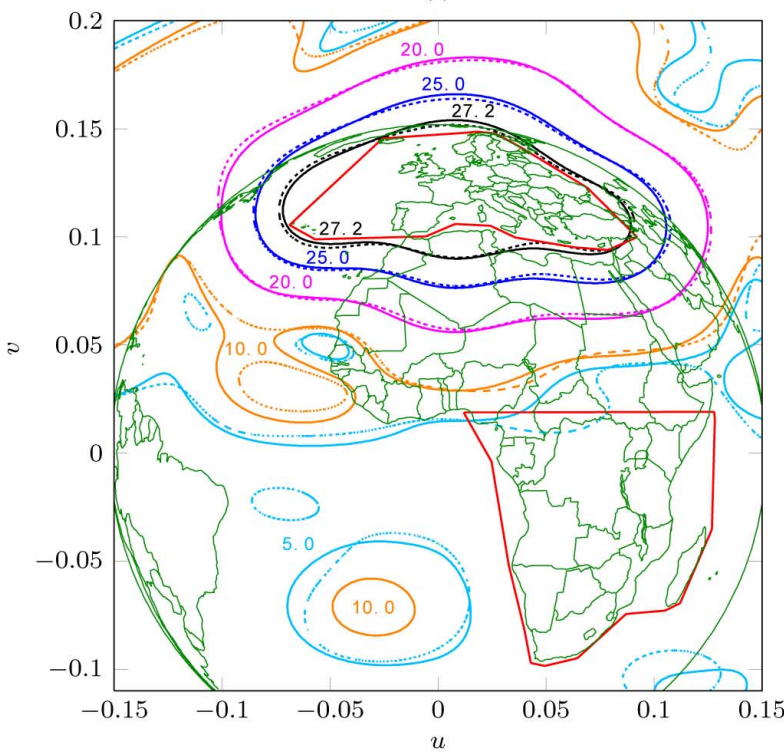

(c)

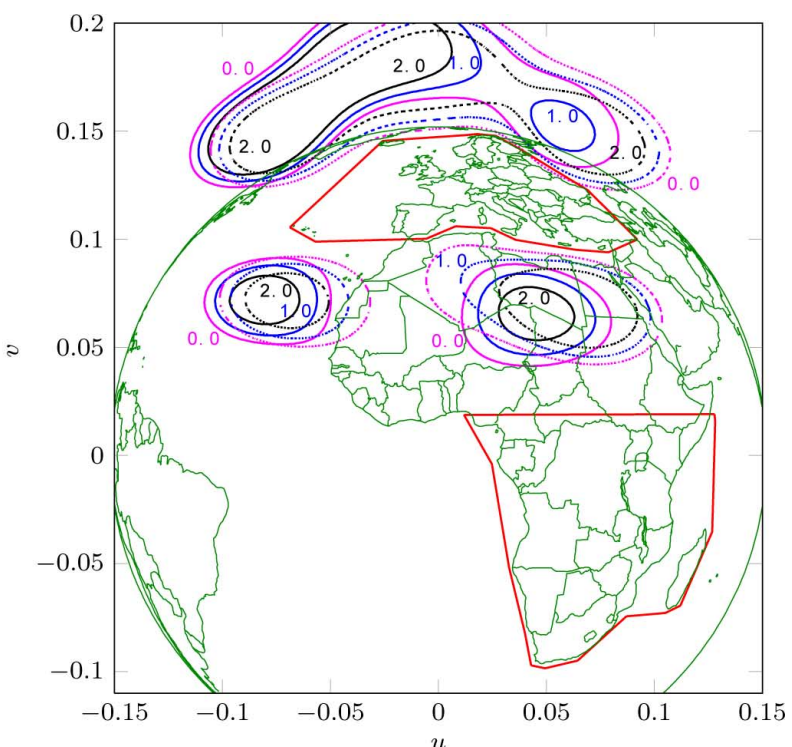

(b)

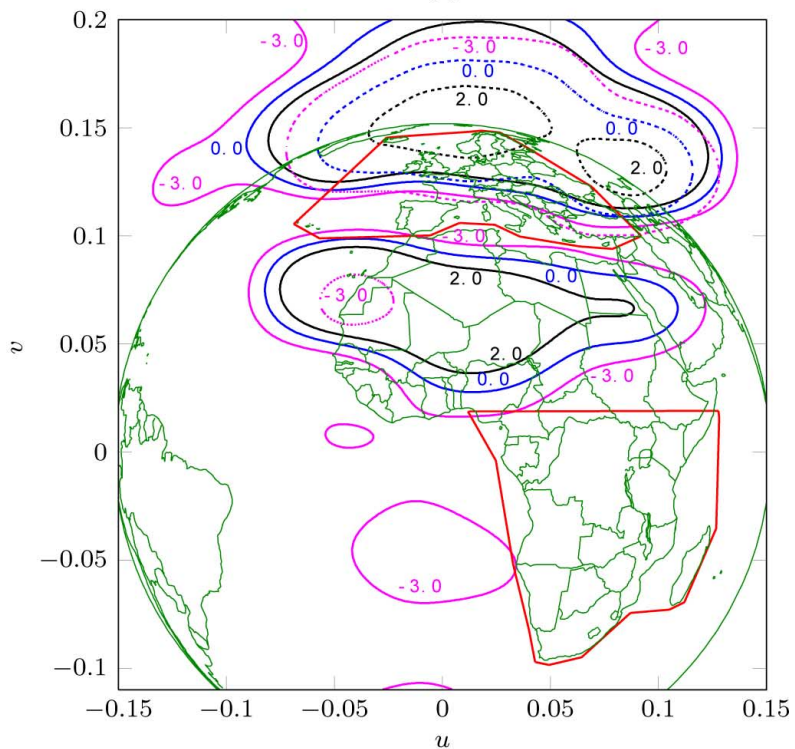

(d)

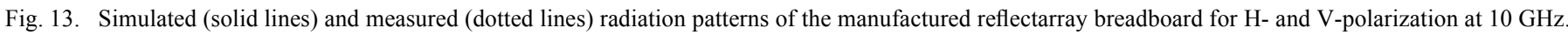

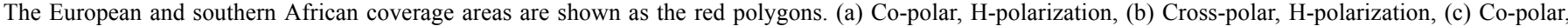
V-polarization, (d) Cross-polar, V-polarization.

within the European coverage area and a minimum isolation (Europe/Africa) level of $27.2 \mathrm{~dB}$. In addition, the cross-polar radiation has been suppressed to below $0 \mathrm{dBi}$, yielding a minimum XPD of $27.8 \mathrm{~dB}$.

Design $\mathrm{C}$ has been compared to a similar reflectarray with regularly positioned elements that has been optimized for the same goals as Design C. The comparison showed an improvement of $1 \mathrm{~dB}$ in the XPD level for Design C, indicating that a better performance in the cross-polar radiation can be obtained by using an irregular array instead of a regular one. However, this improvement is small and is obtained for a single polarization design. Reflectarrays with irregularly and regularly positioned elements optimized for both V-and H-polarizations have also been designed and compared with the aim to investigate the performance for dual-polarization applications. Good results for both polarizations can be obtained with a regular array, while with the grid distortions and square patches used in this work, no further improvement is gained by using an irregular array.

The distortions used in this work are based on Chebyshev polynomials of different orders. These polynomials do not depend nor imitate any of the physical behaviors of the reflectarray and are thus purely mathematical. In order to fully exploit the potential of the irregularity of the element positions, other types of distortions that utilizes the physics of the antenna should to be investigated. A circular or elliptical grid distortion that for instance follows the feed illumination taper over the reflectarray surface could be an example. Such a distortion can be realized by using Zernike polynomials and can be readily included in the GDOT. This is subject to future work. 
TABLE IV

Measured Versus Simulated Data at $10 \mathrm{GHz}$

\begin{tabular}{lcccc}
\hline \hline & $\begin{array}{c}\text { Peak } \\
\text { directivity } \\
(\mathrm{dBi})\end{array}$ & $\begin{array}{c}\text { Min. } \\
\text { directivity } \\
(\mathrm{dBi})\end{array}$ & $\begin{array}{c}\text { Min. } \\
\text { XPD } \\
(\mathrm{dB})\end{array}$ & $\begin{array}{c}\text { Min. } \\
\text { isolation } \\
(\mathrm{dB})\end{array}$ \\
\hline Measurement $(\mathrm{H})$ & 29.2 & 27.3 & 27.2 & 24.3 \\
Simulation $(\mathrm{H})$ & 29.2 & 27.3 & 27.8 & 27.2 \\
\hline Measurement $(\mathrm{V})$ & 29.4 & 27.1 & 24.5 & 20.2 \\
Simulation $(\mathrm{V})$ & 29.4 & 27.1 & 21.0 & 20.5 \\
\hline \hline
\end{tabular}

\section{VALidATION BY MEASUREMENTS}

To verify the accuracy of the GDOT, Design C has been manufactured at DTU and measured at the DTU-ESA Spherical Near-Field Antenna Test Facility. The reflectarray breadboard was measured at a series of frequencies from $9.6 \mathrm{GHz}$ to $10.5 \mathrm{GHz}$ in both $\mathrm{V}$ - and H-polarizations. For the peak directivity, the measurements have a $1 \sigma$ uncertainty of $0.05 \mathrm{~dB}$.

To account for the presence of the support structures, the scattering from the struts is included in the analysis using the MoM add-on in GRASP [35].

In Fig. 13, the simulated (solid lines) and measured (dotted lines) radiation patterns for $\mathrm{H}$ - and $\mathrm{V}$-polarizations at $10 \mathrm{GHz}$ are shown. As expected, the reflectarray has a low co-polar radiation within the southern African contour and a low cross-polar radiation within the European coverage area in H-polarization. A comparison of the solid and dotted lines shows an extremely good agreement between simulations and measurements. The performance of the breadboard is summarized in Table IV, and it is seen that the peak directivity and minimum directivity within the European coverage area are very well predicted, even though the breadboard is based on an irregular grid. For the cross-polar radiation, which is approximately $30 \mathrm{~dB}$ below the co-polar peak, some discrepancies between simulations and measurements can be observed. These discrepancies are expected as the cross-polar level is low and the errors introduced by the assumptions in the LP-SDMoM come into play. Nevertheless, many of the cross-polar radiation features are predicted as seen in Fig. 13(b).

The breadboard was only optimized for H-polarization, hence the lower minimum XPD and isolations levels in V-polarization. The accuracy in the other measured frequencies is also very good, where the maximum discrepancy in the minimum directivity within the European coverage area is $0.1 \mathrm{~dB}$.

These good agreements between simulations and measurements clearly verifies the accuracy of the GDOT.

\section{CONCLUSION}

An accurate and efficient generalized direct optimization technique (GDOT) for the design of printed reflectarrays using arbitrary element shape with irregular orientation and position is presented. It is based on the spectral domain method of moments (SDMoM) assuming local periodicity (LP) and a minimax optimization algorithm. The geometrical parameters of the array elements, i.e., size, orientation, and position, are directly optimized to fulfill the far-field requirements. The optimization uses scattering matrices which are calculated in advance, stored in a look-up table, and accessed during the optimization by a local cubic interpolation. Both co- and cross-polar radiation can be optimized for multiple frequencies, polarizations, and feed illumi- nations. The design procedure has been described and the accuracy of the LP-SDMoM for the design of reflectarrays with irregularly positioned and oriented array elements has been verified by comparisons with full wave method of moments solutions. It is shown that the LP-SDMoM is accurate.

To show the capabilities of the GDOT, three offset contoured beam reflectarrays forming a high-gain beam on a European coverage area have been designed: a linearly polarized broadband design; a circularly polarized design using the variable rotation technique; and a linearly polarized design with irregularly positioned array elements. The latter has been manufactured at the Technical University of Denmark (DTU) and measured at the DTU-ESA Spherical Near-Field Antenna Test Facility. A very good agreement between simulated and measured patterns is obtained, thus verifying the accuracy of the GDOT.

\section{ACKNOWLEDGMENT}

The authors would like to thank Dr. S. Pivnenko, Electromagnetic Systems, Department of Electrical Engineering, Technical University of Denmark (EMS-DTU), for the accurate measurements of the reflectarray breadboard, T. G. Smith (EMSDTU) for valuable comments, and the mechanical workshop at EMS-DTU for the manufacturing of the reflectarray breadboard.

\section{REFERENCES}

[1] J. Huang and J. A. Encinar, Reflectarray Antennas. Piscataway, NJ, USA: IEEE Press, 2008.

[2] H. Legay, D. Bresciani, E. Girard, R. Chiniard, E. Labiole, O. Vendier and G. Caille, "Recent developments on reflectarray antennas at Thales Alenia space," in Proc. EuCAP, Berlin, Germany, 2009.

[3] A. Roederer, "Reflectarray antennas," presented at the EuCAP, Berlin, Germany, 2009.

[4] D. G. Gonzalez, G. E. Pollon, and J. F. Walker, "Microwave Phasing Structures for Electromagnetically Emulating Reflective Surfaces and Focusing Elements for Selected Geometry," U.S. Patent 4905 014, Feb. 27, 1990.

[5] D. M. Pozar, S. D. Targonski, and H. D. Syrigos, "Design of millimeter wave microstrip reflectarrays," IEEE Trans. Antennas Propag., vol. 45, no. 2, pp. 287-296, 1997.

[6] M. R. Chaharmir, J. Shaker, N. Gagnon, and D. Lee, "Design of broadband, single layer dual-band large reflectarray using multi open loop elements," IEEE Trans. Antennas Propag., vol. 58, no. 9, pp. 2875-2883, 2010.

[7] M. E. Bialkowski and K. H. Sayidmarie, "Investigations into phase characteristics of a single-layer reflectarray employing patch or ring elements of variable size," IEEE Trans. Antennas Propag., vol. 56, no. 11, pp. 3366-3372, 2008

[8] L. Moustafa, R. Gillard, F. Peris, R. Loison, H. Legay, and E. Girard, "The phoenix cell: a new reflectarray cell with large bandwidth and rebirth capabilities," IEEE Antennas Wireless Propag. Lett., vol. 10, pp. 71-74, 2011.

[9] M. Bozzi, S. Germani, and L. Perregrini, "Performance comparison of different element shapes used in printed reflectarrays," IEEE Antennas Wireless Propag. Lett., vol. 2, no. 1, pp. 219-222, 2003.

[10] J. Huang and R. J. Pogorzelski, "A ka-band microstrip reflectarray with elements having variable rotation angles," IEEE Trans. Antennas Propag., vol. 46, no. 5, pp. 650-656, 1998.

[11] B. Strassner, C. Han, and K. Chang, "Circularly polarized reflectarray with microstrip ring elements having variable rotation angles," IEEE Trans. Antennas Propag., vol. 52, no. 4, pp. 1122-1125, 2004.

[12] D. Kurup, M. Himdi, and A. Rydberg, "Design of an unequally spaced reflectarray," IEEE Antennas Wireless Propag. Lett., vol. 2, pp. 33-35, 2003.

[13] A. Capozzoli, C. Curcio, A. Liseno, M. Migliorelli, and G. Toso, "Power pattern synthesis of advanced flat aperiodic reflectarrays," presented at the 33rd ESA Antenna Workshop, Noordwijk, Netherlands, 2011.

[14] A. Capozzoli, C. Curcio, G. D’Eila, A. Liseno, G. Toso, and P. Vinetti, "Aperiodic and Non-Planar Array of Electromagnetic Scatterers, and Reflectarray Antenna Comprising the Same," US 20120268340 A1, Sep. 16, 2009. 
[15] M. Zhou, S. B. Sørensen, O. S. Kim, E. Jørgensen, P. Meincke, and O. Breinbjerg, "Direct optimization of printed reflectarrays for contoured beam satellite antenna applications," IEEE Trans. Antennas Propag., vol. 61, no. 4, pp. 1995-2004, 2013.

[16] M. Zhou, S. B. Sørensen, P. Meincke, E. Jørgensen, O. S. Kim, O. Breinbjerg, and G. Toso, "An accurate and efficient design tool for large contoured beam reflectarrays," presented at the ESA Workshop on Large Deployable Antennas, Noordwijk, Netherlands, 2012.

[17] J. A. Encinar, L. S. Datashvili, J. A. Zornoza, M. Arrebola, M. SierraCastaner, J. L. Besada-Sanmartin, H. Baier, and H. Legay, "Dual-polarization dual-coverage reflectarray for space applications," IEEE Trans. Antennas Propag., vol. 54, no. 10, pp. 2827-2837, 2006.

[18] H. Legay, D. Bresciani, E. Labiole, R. Chiniard, E. Girard, G. Caille, L. Marnat, D. Calas, R. Gillard, and G. Toso, "A 1.3 m earth deck reflectarray for a ku band contoured beam antenna," presented at the 33rd ESA Antenna Workshop, Noordwijk, Netherlands, 2011.

[19] J. A. Encinar, M. Arrebola, L. D. L. Fuente, and G. Toso, "A transmitreceive reflectarray antenna for direct broadcast satellite applications," IEEE Trans. Antennas Propag., vol. 59, no. 9, pp. 3255-3264, 2011.

[20] DTU-ESA Spherical Near-Field Antenna Test Facility [Online]. Available: http://www.dtu.dk/centre/ems/English/research/facilities.aspx.

[21] POS, User's Manual. TICRA Engineering Consultants 2011.

[22] CHAMP, User's Manual. TICRA Engineering Consultants 2011.

[23] J. Hald and K. Madsen, "Combined LP and quasi-Newton methods for minimax optimization," Math. Programming, vol. 20, pp. 49-62, 1981.

[24] M. Zhou, S. B. Sørensen, E. Jørgensen, P. Meincke, O. S. Kim, and O. Breinbjerg, "An accurate technique for calculation of radiation from printed reflectarrays," IEEE Antennas Wireless Propag. Lett., vol. 10, pp. 1081-1084, 2011.

[25] R. F. Harrington, Time-Harmonic Electromagnetic Fields. New York, NY, USA: McGraw-Hill, Inc., 1961.

[26] E. Jørgensen, J. Volakis, P. Meincke, and O. Breinbjerg, "Higher order hierarchical Legendre basis functions for electromagnetic modeling," IEEE Trans. Antennas Propag., vol. 52, no. 11, pp. 2985-2995, 2004.

[27] S. R. Rengarajan, "Choice of basis functions for accurate characterization of infinite array of microstrip reflectarray elements," IEEE Antennas Wireless Propag. Lett., vol. 4, pp. 47-50, 2005.

[28] M. Zhou, E. Jørgensen, O. S. Kim, S. B. Sørensen, P. Meincke, and O. Breinbjerg, "Accurate and efficient analysis of printed reflectarrays with arbitrary elements using higher-order hierarchical Legendre basis functions," IEEE Antennas Wireless Propag. Lett., vol. 11, pp. 814-817, 2012.

[29] M. Abramowitz and I. A. E. Stegun, Eds., Handbook of Mathematical Functions 9th ed. New York, NY, USA, Dover, 1970.

[30] J. E. E. Hansen, Spherical Near-Field Antenna Measurements. New York, NY, USA: Peter Peregrinus Ltd, 1988.

[31] Y. Chow, J. Yang, D. Fang, and G. Howard, "A closed-form spatial green's function for the thick microstrip substrate," IEEE Trans. Microwave Theory Tech., vol. 39, no. 3, pp. 588-592, 1991.

[32] Q.-Y Li , Y.-C. Jiao, and G. Zhao, "A novel microstrip rectangularpatch/ring-combination reflectarray element and its application," IEEE Antennas Wireless Propag. Lett., vol. 8, pp. 1119-1122, 2009.

[33] T. G. Smith, N. V. Larsen, U. V. Gothelf, O. S. Kim, and O. Breinbjerg, "20/30 GHZ dual-band circularly polarized reflectarray antenna based on the concentric split loop element," presented at the 34th ESA Antenna Workshop, Noordwijk, Netherlands, 2012.

[34] L. Li, Q. Chen, Q. Yuan, K. Sawaya, T. Maruyama, T. Furuno, and S. Uebayashi, "Novel broadband planar reflectarray with parasitic dipoles for wireless communication applications," IEEE Antennas Wireless Propag. Lett., vol. 8, pp. 881-885, 2009.

[35] K. E. Pontoppidan, GRASP, Technical Description, TICRA Engineering Consultants, 2008.

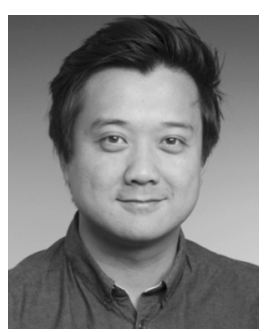

Min Zhou (S'12-M'13) was born in 1984 in Beijing, China. He received the M.Sc. degree in 2009 in electrical engineering and the Ph.D. degree in 2013 from the Technical University of Denmark (DTU), Lyngby, Denmark.

In Fall 2007, he spent five months at the University of Illinois, Urbana-Champaign, IL, USA. Since 2009, he has been with the Danish company TICRA, Copenhagen, Denmark, where he has mainly worked on the analysis and optimization of printed reflectarrays. His research interest include computational electromagnetics, antenna theory, and analysis and design techniques for printed reflectarrays.

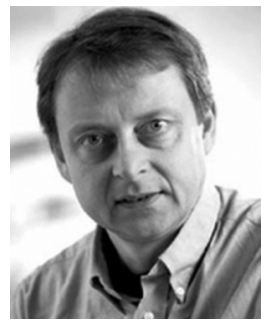

Stig B. Sørensen was born in Denmark, in 1958. $\mathrm{He}$ received the M.Sc. degree and the Industrial Research Education degree from the Technical University of Denmark (DTU), Lyngby, Denmark, in 1982 and 1985, respectively.

Since 1983, he has been with TICRA, Copenhagen, Denmark, where he has been involved in the development of most of the TICRA software packages and the associated numerical electromagnetic methods. His research interest include computational electromagnetics, quasi optical systems, and analysis and synthesis of shaped reflector antennas and printed reflectarrays.

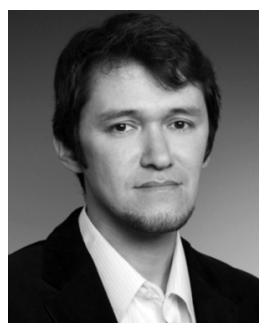

Oleksiy S. Kim received the M.Sc. and Ph.D. degrees from the National Technical University of Ukraine, Kiev, in 1996 and 2000, respectively, both in electrical engineering.

In 2000, he joined the Antenna and Electromagnetics Group at the Technical University of Denmark (DTU), Lyngby, Denmark. He is currently an Associate Professor with the Department of Electrical Engineering, Electromagnetic Systems, DTU. His research interests include computational electromagnetics, metamaterials, electrically small antennas, photonic bandgap, and plasmonic structures.

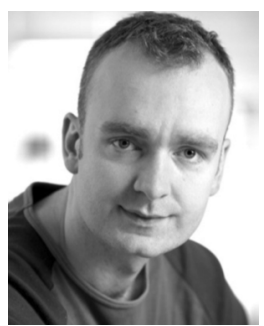

Erik Jørgensen (S'98-M'03) was born in 1974 in Denmark. He received the M.Sc. and Ph.D. degrees in electrical engineering from the Technical University of Denmark (DTU), Lyngby, Denmark, in 2000 and 2003, respectively.

In Fall 1998, he spent five months at the University of Siena, Italy. In Fall 2001, he was a Visiting Scholar at the Radiation Laboratory, University of Michigan, Ann Arbor, MI, USA. In 2003, he joined the Danish company TICRA, Copenhagen, where he is now leading the development of commercial software for reflector antenna analysis and antenna diagnostics. His research interests include computational electromagnetics and high-frequency techniques.

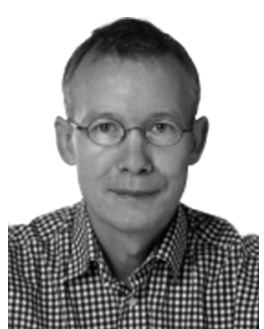

Peter Meincke (S'93-M'96) was born in Roskilde, Denmark, on November 25, 1969. He received the M.Sc. and Ph.D. degrees in electrical engineering from the Technical University of Denmark (DTU), Lyngby, Denmark, in 1993 and 1996, respectively.

In spring and summer of 1995, he was a Visiting Research Scientist at the Electromagnetics Directorate of Rome Laboratory, Hanscom Air Force Base, MA, USA. In 1997, he was with a Danish cellular phone company, working on theoretical aspects of radio-wave propagation. In spring and summer of 1998, he was visiting the Center for Electromagnetics Research, Northeastern University, Boston, MA, USA, while holding a Postdoctoral position from DTU. In 1999, he became a staff member of the Department of Electromagnetic Systems, DTU. He was an Associate Professor with Ørsted-DTU, Electromagnetic Systems, DTU, where his teaching and research interests included electromagnetic theory, inverse problems, high-frequency and time-domain scattering, antenna theory, and microwave imaging. Since 2008, he has been with TICRA, Copenhagen, Denmark, where he is currently developing software for reflector antenna analysis and antenna diagnostics.

Dr. Meincke won the first prize award in the 1996 IEEE Antennas and Propagation Society Student Paper Contest in Baltimore, MD, USA, for his paper on uniform physical theory of diffraction equivalent edge currents and received the R.W.P. King Paper Award in 2000 for his paper "Time-domain version of the physical theory of diffraction" published in IEEE TRANSACTIONS ON ANTENNAS AND PROPAGATION, February, 1999. 


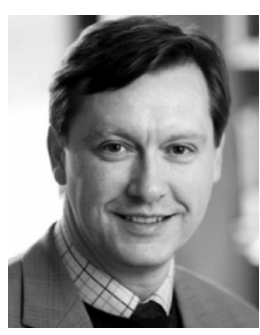

Olav Breinbjerg ( $\left.M^{\prime} 87\right)$ was born in Silkeborg, Denmark, in 1961. He received the M.Sc. and Ph.D. degrees in electrical engineering from the Technical University of Denmark (DTU), Lyngby, Denmark, in 1987 and 1992, respectively.

Since 1991, he has been on the Faculty of the Department of Electrical Engineering as Assistant Professor from 1991 to 1995, Associate Professor from 1995 to 2005, and Full Professor since 2006. He is now Head of the Electromagnetic Systems Group and the DTU-ESA Spherical Near-Field Antenna Test Facility. He was a Visiting Scientist at Rome Laboratory in 1988, a Fulbright Research Scholar at the University of Texas at Austin in 1995, and a Visiting Professor at the University of Siena in 2011. He has been, or is, the main supervisor of $12 \mathrm{Ph} . \mathrm{D}$. projects. His research is generally in applied electromagnetics - and particularly in antennas, antenna measurements, computational techniques, and scattering - for applications in wireless communication and sensing technologies. He is the author or coauthor of more than 50 journal papers, 150 conference papers, and 75 technical reports.

Dr. Breinbjerg received a U.S. Fulbright Research Award in 1995. He also received the 2001 AEG Elektron Foundation's Award in recognition of his research in applied electromagnetics and the 2003 DTU Student Union's Teacher of the Year Award for his course on electromagnetics.

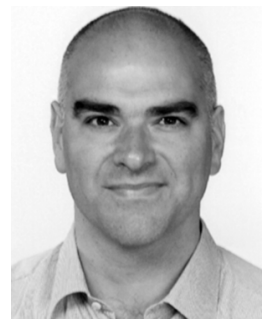

Giovanni Toso (S'93-M'00-SM'07) was born in La Spezia, Italy, on May 3, 1967. He received the Laurea Degree (summa cum laude) and the Ph.D. degree in electrical engineering from the University of Florence, Florence, Italy, in 1992 and 1995 respectively.

In 1996 he was visiting scientist at the Laboratoire d'Optique Electromagnétique, University of Aix-Marseille III, Marseille, France. From 1997 to 1999 he was a Post Doctoral student at the University of Florence. In 1999 he was a visiting scientist at the University of California, Los Angeles (UCLA), CA, USA. In the same year he received a scholarship from Thales Alenia Space, Rome, Italy, and he has been appointed researcher in a Radioastronomy Observatory of the Italian Nationa Council of Researches (CNR). Since 2000 he is with the Antenna and Submillimeter Section of the European Space and Technology Centre of the European Space Agency, ESA ESTEC, Noordwijk, The Netherlands. His research interests are mainly in the field of array antennas for satellite applications and, in particular, in non regular arrays, reflectarrays and multibeam antennas. In 2009 he has been coeditor of the Special Issue on Active Antennas for Satellite Applications in the International Journal of Antennas and Propagation. 